\title{
Determination of reference genes for circadian studies in different tissues and mouse strains
}

\author{
Rok Kosir ${ }^{1}$, Jure Acimovic ${ }^{2}$, Marko Golicnik², Martina Perse ${ }^{3}$, Gregor Majdic $^{4}$, Martina Fink ${ }^{5}$, Damjana Rozman $^{1 *}$
}

\begin{abstract}
Background: Circadian rhythms have a profound effect on human health. Their disruption can lead to serious pathologies, such as cancer and obesity. Gene expression studies in these pathologies are often studied in different mouse strains by quantitative real time polymerase chain reaction (qPCR). Selection of reference genes is a crucial step of qPCR experiments. Recent studies show that reference gene stability can vary between species and tissues, but none has taken circadian experiments into consideration.

Results: In the present study the expression of ten candidate reference genes (Actb, Eif2a, Gapdh, Hmbs, Hprt1, Ppib, Rn18s, Rplp0, Tbcc and Utp6c) was measured in 131 liver and 97 adrenal gland samples taken from three mouse strains (C57BL/6JOlaHsd, 129Pas plus C57BL/6J and Crem KO on 129Pas plus C57BL/6J background) every $4 \mathrm{~h}$ in a $24 \mathrm{~h}$ period. Expression stability was evaluated by geNorm and NormFinder programs. Differences in ranking of the most stable reference genes were observed both between individual mouse strains as well as between tissues within each mouse strain. We show that selection of reference gene (Actb) that is often used for analyses in individual mouse strains leads to errors if used for normalization when different mouse strains are compared. We identified alternative reference genes that are stable in these comparisons.

Conclusions: Genetic background and circadian time influence the expression stability of reference genes. Differences between mouse strains and tissues should be taken into consideration to avoid false interpretations. We show that the use of a single reference gene can lead to false biological conclusions. This manuscript provides a useful reference point for researchers that search for stable reference genes in the field of circadian biology.
\end{abstract}

\section{Background}

Circadian rhythms are oscillations in behaviour and physiology whose function it is to anticipate environmental changes associated with the solar day [1]. At the molecular level, they consist of a network of transcriptional and translational feedback loops that drive the $24 \mathrm{~h}$ expression of core clock components [2,3]. Circadian control is required for healthy life, thus disruption of circadian cycle leads to pathologies such as cancer, obesity, lipid disorders and type 2-diabetes [4-6]. Some of these abnormalities were discovered in mouse models lacking core clock genes Clock and Bmal1 [7-9]. Phenotypes resulting from mutations of clock genes are highly affected by genetic background $[4,10,11]$. Yoshiki

\footnotetext{
* Correspondence: damjana.rozman@mf.uni-lj.si

${ }^{1}$ Center for Functional Genomics and Bio-Chips, Institute of Biochemistry, Faculty of Medicine, University of Ljubljana, Zaloska 4, SI-1000 Ljubljana, Slovenia

Full list of author information is available at the end of the article
}

defined genetic background as the influence of all genes of the genome that may interact with the gene of interest and potentially influence the specific phenotype [12]. To date, a number of reports have shown that genetic background affects the phenotype caused by gene disruption [12-15].

When studying gene expression, qPCR is the dominant quantitative technique due to its broad dynamic range, accuracy, sensitivity, specificity and speed [16]. Normalization in qPCR controls for variations in all experimental steps, enabling comparison between different samples [17]. Different normalization strategies are available, where application of reference genes as internal controls seems to be the most appropriate $[18,19]$. Unfortunately, there is no universal reference gene that would be stably expressed under all experimental conditions. Hence, normalization to reference genes that are validated in individual experiments is a prerequisite for accurate interpretation of biological data [20-24]. 
The broader scope of our research is to understand the circadian expression of genes in different tissues of the two commonly used laboratory mouse strains (C57BL/6JOlaHsd and 129Pas plus C57BL/6J) and to determine the tissue-specific effect of the targeted disruption of transcription factor Crem. In this manuscript, we show that genetic background and the circadian time are important factors influencing expression of commonly used qPCR reference genes. This should be taken into consideration for accurate interpretation of biological data.

\section{Results}

Selection and characteristics of candidate reference genes Seven candidate reference genes (Ppib, Rplp0, Gapdh, Actb, Hmbs, Hprt1 and Rn18s) were selected [20,23] and their expression measured in 35 livers and 33 adrenals from the inbred strain (C57BL/6JOlaHsd), 51 livers and 34 adrenals from the wild type mixed strain (129Pas plus C57BL/6J) and 45 livers and 30 adrenals from the mixed strain with a targeted disruption of the Crem gene (Crem $\mathrm{KO})$. For livers, three additional reference genes were selected by RefGenes (Eif2a, Utp $6 c$ and Tbcc), based on meta analysis of the most stably expressed liver genes in various mouse strains as detected by Affymetrix chips [25]. Gene symbols, full names, accession numbers and gene functions are listed in Table 1 . Intron spanning primers were designed wherever possible, with the exception of $R p l p O$ and $T b c c$ where primers lie within a single exon. To determine primer specificity, melting curve analyses were performed on all primer pairs during the primer validation process as well as after each qPCR run. The specificity of the amplicon was confirmed by the presence of a single peak. Primer efficiencies were calculated based on slopes from standard curves by LightCycler 480 software (Roche Diagnostics). Standard curves were prepared with five-fold serial dilutions of the cDNA pool. A negative control (without reverse transcriptase) was also included to determine possible amplification from genomic DNA. Only primers with single peaks and good negative controls were used in the study. Primer details are listed in Table 2.

\section{Expression level of reference genes}

Expression of measured reference genes is represented as raw quantification cycle $(\mathrm{Cq})$ in Figure 1. $\mathrm{Rn} 18 \mathrm{~s}$ shows highest expression in all samples with a mean $\mathrm{Cq}$ of 8.8. This is not surprising since $R n 18 \mathrm{~s}$ represents the bulk of total RNA. The Cq values of other candidates were between $\mathrm{Cq}$ 18-29. Tbcc shows lowest expression in all samples with a mean $\mathrm{Cq}$ of 28.5. The largest variation across the studied 228 samples was observed in Actb and Hmbs (12 cycles) and the smallest for Eif $2 a$, Tbcc and Utp6c (< 4 cycles).

\section{Search for optimal reference genes by geNorm}

geNorm ranks reference genes according to their average expression stability value (M), from the most stable (lowest $M$ value) to the least stable (highest $M$ value). An important advantage of geNorm is that it provides the optimal number of reference genes required for accurate normalization. This number is based on the pairwise variation values $\left(\mathrm{V}_{(\mathrm{n} / \mathrm{n}+1)}\right)$ [20].

We divided expression data into eight groups, according to the tissue (liver or adrenal) and mouse strain (C57BL/6JOlaHsd; 129Pas plus C57BL/6J; CremKO in mixed strain or including all strains; Table 3). Groups A to $F$ thus represent stably expressed reference genes of each tissue in each mouse strain. Groups $\mathrm{G}$ and $\mathrm{H}$ consider genetic differences between mouse strains because samples of all strains are joined for each tissue. The time of sacrifice $\left(C_{t}\right)$ cannot be included as a variable by geNorm analyses. The same groups have been applied as well in NormFinder analyses.

Figure 2 summarises the average expression stabilities and ranking of candidate reference genes in individual

Table 1 Official symbols, accession numbers, full names and functions of candidate reference genes evaluated in this study

\begin{tabular}{llll}
\hline Symbol & $\begin{array}{l}\text { Accession } \\
\text { number }\end{array}$ & Full name & Function \\
\hline Rplp0 & NM_007475.4 & ribosomal protein, large, P0 & Structural constituent of ribosome \\
Ppib & NM_011149.2 & peptidylprolyl isomerase B & Associated with the secretory pathway and released in \\
& & biological fluids & Cytoskeletal structural protein \\
Actb & NM_007393.3 & actin, beta & Heme synthesis, porphyrin metabolism \\
Hmbs & NM_013551.2 & Hydroxymethyl-bilane synthase & Purine synthesis in salvage pathway \\
Hprt1 & NM_013556.2 & hypoxanthine guanine phosphoribosyl transferase 1 & Ribosomal RNA \\
Rn18s & NR_003278.1 & 18 S RNA & Protein translation \\
Eif2a & NM_001005509.1 & eukaryotic translation initiation factor 2a & Rn18 s biogenesis \\
Utp6c & NM_144826.3 & small subunit (SSU) processome component, homolog & (yeast) \\
& & tubulin-specific chaperone c & Protein folding \\
\hline Tbcc & NM_178385.3 & &
\end{tabular}


Table 2 Primer sequences, exon location (where possible), efficiency and amplicon length for candidate reference genes and the test circadian gene

\begin{tabular}{|c|c|c|c|c|c|}
\hline Symbol & Primer & Sequence & Amplicon length & Primer location ${ }^{1}$ [exon] & Primer efficiency \\
\hline \multicolumn{6}{|c|}{ Reference genes } \\
\hline \multirow[t]{2}{*}{ Rplpo } & fw & CACTGGTCTAGGACCCGAGAAG & 73 & 4 & 1.98 \\
\hline & rv & GGTGCCTCTGGAGATTTTCG & & 4 & \\
\hline \multirow[t]{2}{*}{ Ppib } & fw & GGAGATGGCACAGGAGGAAA & 73 & 3 & 1.93 \\
\hline & rv & CCGTAGTGCTTCAGTTTGAAGTTCT & & 4 & \\
\hline \multirow[t]{2}{*}{ Gapdh } & fw & CCAATGTGTCCGTCGTGGATCT & 239 & 5 & 1.94 \\
\hline & rv & GTTGAAGTCGCAGGAGACAACC & & 6 & \\
\hline \multirow[t]{2}{*}{ Actb } & fw & CTTCCTCCCTGGAGAAGAGC & 124 & 4 & 1.98 \\
\hline & rv & ATGCCACAGGATTCCATACC & & 5 & \\
\hline \multirow[t]{2}{*}{ Hmbs } & fw & TCCCTGAAGGATGTGCCTA & 73 & 7 & 1.64 \\
\hline & rv & AAGGGTTTTCCCGTTTGC & & 8 & \\
\hline \multirow[t]{2}{*}{ Hprt1 } & fw & TCCTCCTCAGACCGCTTTT & 90 & 1 & 1.89 \\
\hline & rv & СCTGGTTCATCATCGCTAATC & & 2 & \\
\hline \multirow[t]{2}{*}{ Rn18s } & fw & CGCCGCTAGAGGTGAAATTC & 62 & n.a. & 1.79 \\
\hline & rv & TTGGCAAATGCTTTCGCTC & & n.a. & \\
\hline \multirow[t]{2}{*}{ Eif2a } & fw & CAACGTGGCAGCCTTACA & 74 & 2 & 1.95 \\
\hline & rv & TTTCATGTCATAAAGTTGTAGGTTAGG & & 3 & \\
\hline \multirow[t]{2}{*}{ Utp6c } & fw & TTTCGGTTGAGTTTTTCAGGA & 75 & 17 & 1.82 \\
\hline & rv & CCCTCAGGTTTACCATCTTGC & & 18 & \\
\hline \multirow[t]{2}{*}{ Tbcc } & fw & GACTCCTTCCTGAACCTCTGG & 62 & n.a. & 1.90 \\
\hline & rv & GGAGGCCATTCAAAACTTCA & & n.a. & \\
\hline \multicolumn{6}{|c|}{ Circadian gene } \\
\hline \multirow[t]{2}{*}{$\overline{D b p}$} & fw & AATGACCTTTGAACCTGATCCCGCT & 175 & 7 & 1.93 \\
\hline & rv & GCTCCAGTACTTCTCATCCTTCTGT & & 7 & \\
\hline
\end{tabular}

groups. Ranking is different for livers of different mouse strains. In 129Pas plus C57BL/6J and Crem KO, which has the Crem gene disrupted on the same mixed background, Hprt1 is always most stable, together with either

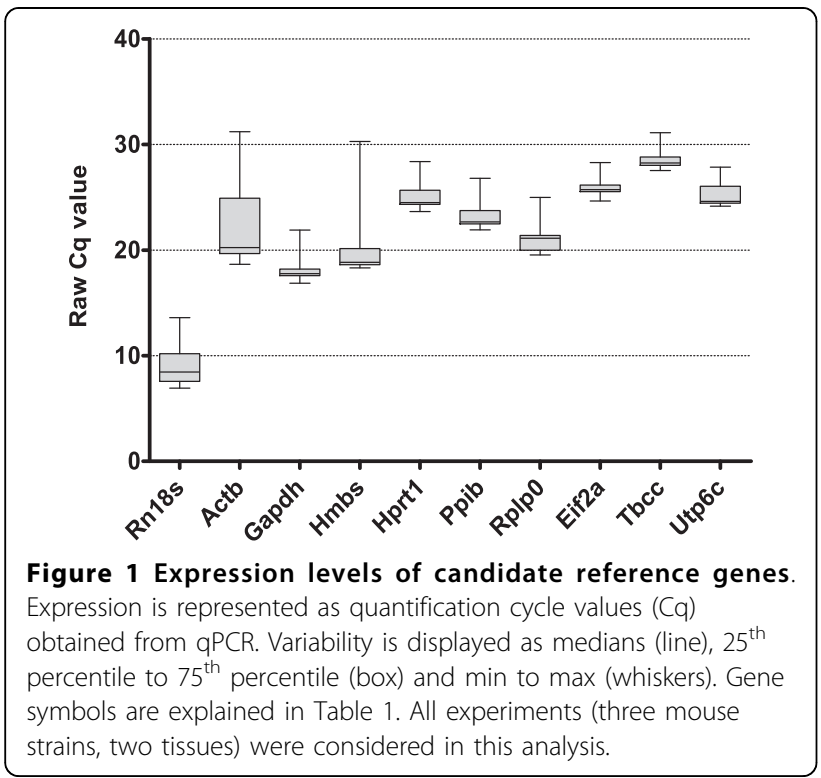

Rn18 s (Figure 2E) or RplpO (Figure 2C). In the C57BL/ 6JOlaHsd strain, the most stable genes are Eif2 $a$ and $H m b s$ (Figure 2A), which were, interestingly, among the least stable in the mixed strain (Figure 2C,2E). In adrenal glands of individual mouse strains, the ranking is more consistent, with Hprt1 and Actb both ranking last and $R n 18 s$ and Ppib always ranking among the top three (Figure 2B,2D,2F).

When evaluating reference genes within each strain, relatively small $M$ values are observed (Figure $2 \mathrm{~A}$ to $2 \mathrm{~F}$ ), indicating a greater degree of expression stability between samples. However, when genetic differences between mouse strains are included in evaluation, a larger degree of variation (larger $\mathrm{M}$ value) is observed (Figure $2 \mathrm{G}$ and $2 \mathrm{H})$, which supports the notion that genetic variability importantly influences expression. In this case, $A c t b$ is the least stable in both liver and adrenal glands. It also displays a far greater $M$ value compared to other genes, showing that it is indeed not a good gene for normalization in mouse tissues. Eif $2 a$ and Tbcc in the liver (Figure 2G) and Gapdh and Hmbs in adrenals (Figure $2 \mathrm{H}$ ) are most stable if all strains are considered.

We also determined the optimal number of reference genes for normalization (Figure 3$)$. In all groups $(A-H)$, a pairwise variation value $(\mathrm{V} 2 / 3)$ of less than 0.15 
Table 3 Distribution of RT-qPCR data into groups

\begin{tabular}{|c|c|c|c|c|}
\hline Mouse Strain & Tissue & Time Point $[\mathrm{Ct}]$ & Biological replicates & $\begin{array}{c}\text { Dataset } \\
\text { [num. of samples] }\end{array}$ \\
\hline \multirow[t]{14}{*}{ C57BL/6JOlaHsd } & Liver & 0 & 5 & $\begin{array}{c}\mathrm{A} \\
{[35]}\end{array}$ \\
\hline & & 4 & 5 & \\
\hline & & 8 & 5 & \\
\hline & & 12 & 5 & \\
\hline & & 16 & 5 & \\
\hline & & 20 & 5 & \\
\hline & & 24 & 5 & \\
\hline & Adrenal gland & 0 & 5 & $\begin{array}{c}B \\
{[33]}\end{array}$ \\
\hline & & 4 & 4 & \\
\hline & & 8 & 5 & \\
\hline & & 12 & 5 & \\
\hline & & 16 & 5 & \\
\hline & & 20 & 5 & \\
\hline & & 24 & 4 & \\
\hline 129Pas plus C57BI/6J & Liver & 0 & 6 & $\begin{array}{c}C \\
{[45]}\end{array}$ \\
\hline \multirow[t]{13}{*}{ Crem knock-out } & & 4 & 6 & \\
\hline & & 8 & 7 & \\
\hline & & 12 & 6 & \\
\hline & & 16 & 6 & \\
\hline & & 20 & 7 & \\
\hline & & 24 & 7 & \\
\hline & Adrenal gland & 0 & 3 & $\begin{array}{c}\mathrm{D} \\
{[30]}\end{array}$ \\
\hline & & 4 & 4 & \\
\hline & & 8 & 4 & \\
\hline & & 12 & 5 & \\
\hline & & 16 & 4 & \\
\hline & & 20 & 5 & \\
\hline & & 24 & 5 & \\
\hline 129Pas plus C57BI/6J & Liver & 0 & 8 & $\begin{array}{c}E \\
{[51]}\end{array}$ \\
\hline \multirow[t]{13}{*}{ Wild type } & & 4 & 7 & \\
\hline & & 8 & 7 & \\
\hline & & 12 & 7 & \\
\hline & & 16 & 7 & \\
\hline & & 20 & 8 & \\
\hline & & 24 & 7 & \\
\hline & Adrenal gland & 0 & 5 & $\begin{array}{c}F \\
{[34]}\end{array}$ \\
\hline & & 4 & 5 & \\
\hline & & 8 & 4 & \\
\hline & & 12 & 5 & \\
\hline & & 16 & 5 & \\
\hline & & 20 & 5 & \\
\hline & & 24 & 5 & \\
\hline All strains & Liver & 7 time points & 131 & G \\
\hline All strains & Adrenal & 7 time points & 97 & $\mathrm{H}$ \\
\hline Total number of sam & & & 228 & \\
\hline
\end{tabular}




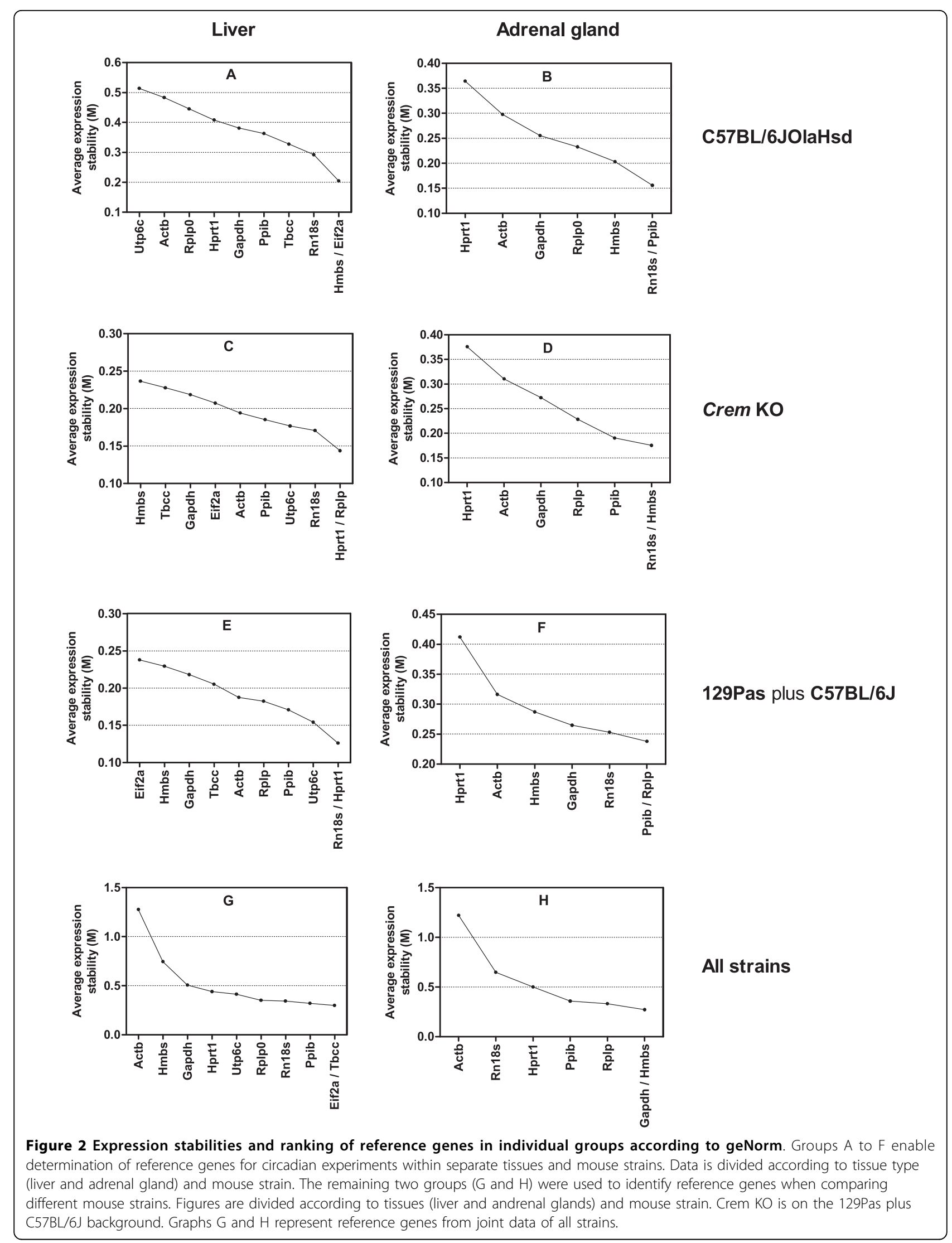


Liver

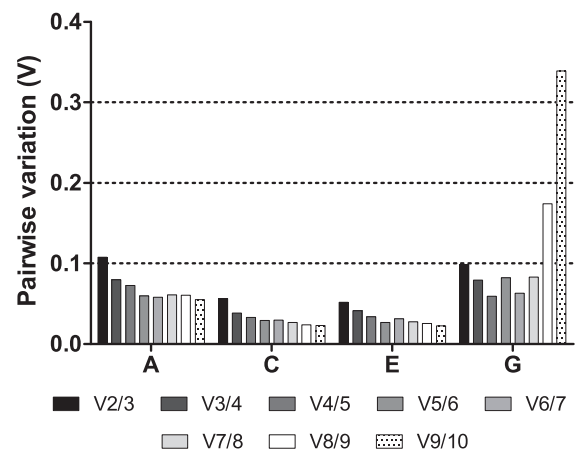

Adrenal gland

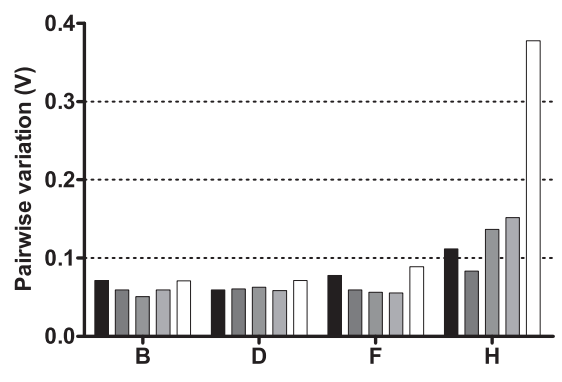

- V2/3 $\square \mathrm{V} 3 / 4 \quad \square \mathrm{V} 4 / 5 \quad \square \mathrm{V} 5 / 6 \quad \square \mathrm{V} 6 / 7$

Figure 3 Optimal number of reference genes used for normalization. geNorm determination of the optimal number of reference genes based on the pairwise variation value $\left(V_{\mathbf{n} / \mathbf{n}+\mathbf{1}}\right)$ that is calculated between two sequential normalization factors [20]. The optimal number of reference genes was calculated for liver and adrenal glands where samples from all mouse strains are included.

was determined, confirming that 2 stable reference genes might be sufficient for accurate normalization, as proposed by Vandesompele et al [20]. Addition of further genes in groups $\mathrm{A}$ to $\mathrm{F}$ did not influence the $\mathrm{V}$ value significantly. However, in groups $\mathrm{G}$ and $\mathrm{H}$, addition of least stable genes did raise the $\mathrm{V}$ value above 0.15 (Figure 3).

\section{Determination of reference genes by NormFinder}

NormFinder uses a model based approach to calculate gene stability value for either the most stable reference gene or the best combination of two genes. It has the advantage to allow estimation of variations between time points, which is crucial in circadian experiments. NormFinder gene stability values and rankings are shown in Table 4. In this analysis, samples in all groups $(\mathrm{A}-\mathrm{H})$ were divided further into 7 subgroups according to the time of scarification (time point).

Similarly to geNorm, NormFinder also showed differences in ranking of reference genes in different mouse strains. In liver samples of $129 \mathrm{Pas}$ plus $\mathrm{C} 57 \mathrm{BL} / 6 \mathrm{~J}$ and Crem KO strains Hprt1 always ranks among the top two reference genes (Table $4 \mathrm{C}$ and $4 \mathrm{E}$ ), however in the C57BL/6JOlaHsd strain it is among the least stable genes (Table 4A). The opposite is seen for Hmbs, which is most stable in the C57BL/6JOlaHsd strain (Table 4A) and among least stable in 129Pas plus C57BL/6J and Crem $\mathrm{KO}$ strains (Table 4C and 4E). When genetic differences between strains are included in the evaluation,

Table 4 Stability value and ranking of reference genes based on NormFinder

\begin{tabular}{|c|c|c|c|c|c|c|c|c|}
\hline \multirow[b]{3}{*}{ Gene } & \multicolumn{8}{|c|}{ Circadian } \\
\hline & \multicolumn{2}{|c|}{ C57BL/6JOlaHsd } & \multicolumn{2}{|c|}{ 129Pas plus C57BI/6J-KO } & \multicolumn{2}{|c|}{ 129Pas plus C57BI/6J-WT } & \multicolumn{2}{|c|}{ Mouse strain } \\
\hline & liver & adrenal gland & liver & adrenal gland & liver & adrenal gland & liver & adrenal gland \\
\hline Dataset & A & B & $\mathrm{C}$ & D & $E$ & $\mathbf{F}$ & G & $\mathrm{H}$ \\
\hline Rn18s & 0.118 & 0.070 & 0.078 & 0.068 & 0.063 & 0.062 & 0.020 & 0.054 \\
\hline Actb & 0.196 & 0.090 & 0.093 & 0.104 & 0.087 & 0.060 & 0.555 & 0.506 \\
\hline Gapdh & 0.134 & 0.088 & 0.123 & 0.113 & 0.106 & 0.058 & 0.112 & 0.080 \\
\hline Hmbs & $0.096^{*}$ & 0.058 & 0.114 & 0.080 & 0.095 & 0.063 & 0.309 & 0.088 \\
\hline Hprt1 & 0.150 & 0.156 & 0.062 & 0.166 & 0.058 & 0.187 & 0.024 & 0.199 \\
\hline Ppib & 0.124 & $0.057^{*}$ & 0.090 & $0.062^{*}$ & 0.070 & $0.025^{*}$ & 0.028 & $0.028^{*}$ \\
\hline Rplp0 & 0.187 & 0.081 & $0.056^{*}$ & 0.099 & 0.077 & 0.073 & 0.021 & 0.064 \\
\hline Eif2a & 0.127 & & 0.112 & & 0.106 & & $0.017^{*}$ & \\
\hline Tbcc & 0.124 & & 0.107 & & 0.081 & & 0.021 & \\
\hline Utp6c & 0.190 & & 0.060 & & $0.057^{*}$ & & 0.027 & \\
\hline \multirow[t]{3}{*}{ Best two } & Ppib & Ppib & Hprt1 & Rn18s & Hprt1 & Gapdh & Rn18s & Rn18s \\
\hline & Hmbs & Hmbs & Ppib & Ppib & Utp6c & Ppib & Eif2a & Ppib \\
\hline & 0.061 & 0.046 & 0.044 & 0.048 & 0.037 & 0.032 & 0.013 & 0.031 \\
\hline
\end{tabular}

In groups $\mathrm{A}$ to $\mathrm{F}$, data was grouped according to tissue, mouse strain and the time point. In groups $\mathrm{G}$ and $\mathrm{H}$, data was grouped only according to tissue and time point. *-best candidate reference gene according to NormFinder. (Best two) - the best combination of two reference genes together with their stability value are calculated only if group identifiers (in our case time of sacrifice) are included in the analysis. "Best two" is not always equal to the first and second ranking genes, but represent the two genes with minimal combined inter-and intragroup expression variation [22]. 
Actb is the least stable gene (Table 4G), followed by Hmbs and Gapdh.

Ranking is again more consistent for adrenal glands. Ppib ranks as the most stable reference gene both in each individual mouse strain (Table 4B,D and 4F) and also irrespective of the mouse genetic background (Table $4 \mathrm{H})$. Hprt1 is the least stable gene in adrenals of each investigated mouse strain (Table 4B, D and 4F), while Actb ranks last. Again, this result is obtained only if genetic differences between strains are taken into consideration (Table $4 \mathrm{H}$ ).

In individual mouse strain groups $(\mathrm{A}-\mathrm{E})$, with the exception of group $\mathrm{F}$, gene stability value for the best combination of two reference genes is lower than the value of the most stable reference gene (Table 4), suggesting that normalization on a single reference gene may not be sufficient.

\section{Normalization of biological data by reference genes of different stability}

$D b p$ (D-box binding protein) is one of the most robust circadian genes in the liver, with the peak of expression between CT10-CT14 [26]. We monitored the Dbp hepatic expression (Cq values) in mouse strains $\mathrm{C} 57 \mathrm{BL} /$ 6JOlaHsd, 129Pas plus C57BL/6J and Crem KO. To test the role of reference gene selection on interpretation of the hepatic $D b p$ expression, we applied different normalization procedures: with a) Actb which is commonly used for normalization, but was determined as the least stable gene by geNorm and NormFinder; b) the average of the two most stable reference genes according to geNorm (Eif2a and Tbcc) and c) the average of the two most stable reference genes according to NormFinder (Rn18 $s$ and Eif2a).

Figure 4 shows that normalization to Actb inserts bias into the data. A seemingly large difference in $D b p$ expression is detected between the C57BL/6JOlaHsd and the 129Pas plus C57BL/6J and Crem KO strains (Figure 4A). This leads to a false conclusion that $D b p$ has a very high expression in the C57BL/6JOlaHsd strain, with almost none in the 129Pas plus C57BL/6J and Crem KO strains. However, when normalization is carried out with the most stable reference genes determined by either geNorm or NormFinder, the difference is greatly reduced (Figure $4 \mathrm{~B}$ and $4 \mathrm{C}$ ). In this case, $D b p$ is equally expressed in both mouse strains, which can be expected for a gene with a high expression level and robust circadian rhythm [27].

\section{Discussion}

qPCR is a method of choice for quantitative gene expression analysis. Due to its high sensitivity, normalization with stable reference genes is important for accurate analysis of the biological variation in the data

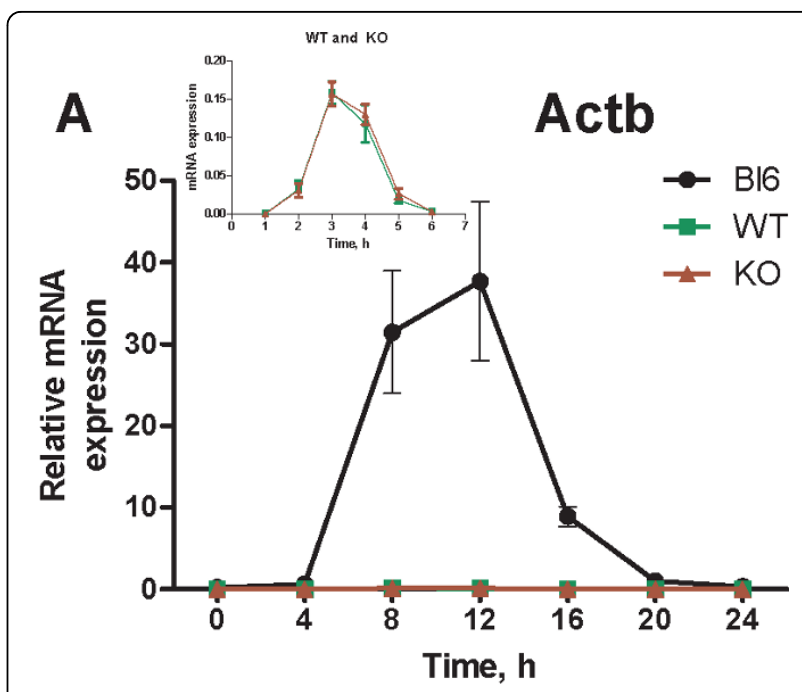

B

Eif2a, Tbcc (geNorm)

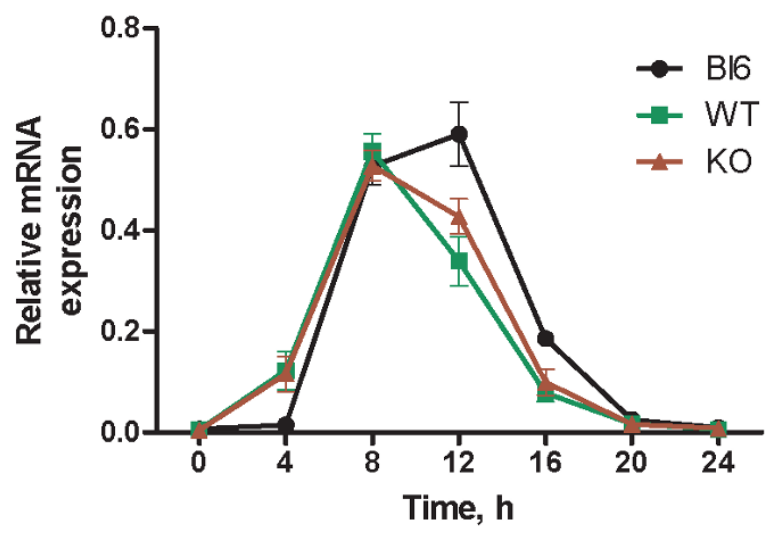

C Rn18s, Eif2a (NormFinder)

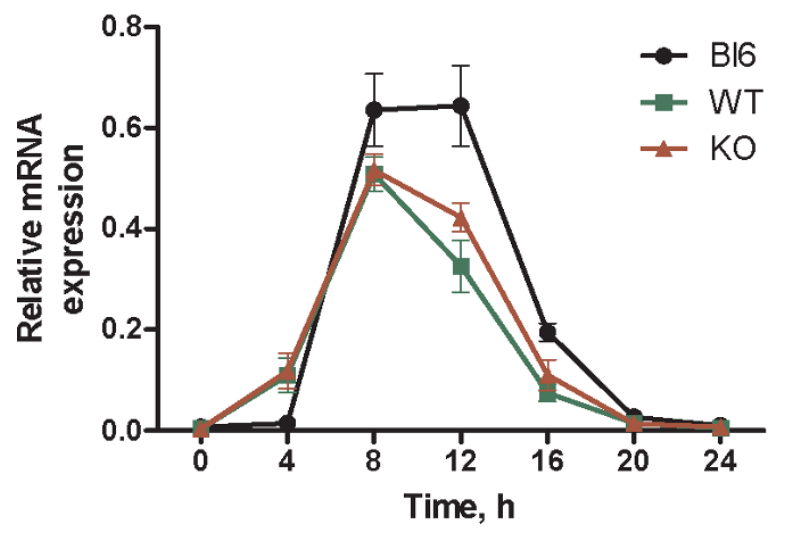

Figure 4 Liver expression profile of the mouse circadian gene $\boldsymbol{D} \boldsymbol{b} \boldsymbol{p}$. The $\boldsymbol{D} \boldsymbol{b} \boldsymbol{p}$ gene with known circadian transcription in mouse liver was normalized with an unstable reference gene $\boldsymbol{A} \boldsymbol{c t} \boldsymbol{b}(\mathrm{A})$ or with the two most stable reference genes as calculated by geNorm (B) or NormFinder (C). Due to low values not seen in panel A, an insert represents the expression profile of $\mathbf{D} \boldsymbol{b} \boldsymbol{p}$ in 129Pas plus C57BL/6J and Crem KO mouse strains. 
$[18,23,28]$. The selection of appropriate reference genes is, however, far from trivial. It has been shown that application of non-validated reference genes can lead to inaccurate data interpretation $[23,29,30]$.

Understanding the tissue-specific circadian behaviour of genes and proteins is often required in drug-treated mouse models, including knockout models from different mouse strains. In recent years, the circadian aspects of metabolism and drug detoxification became more important for proper understanding of physiology, pathophysiology, drug metabolism, etc. [5,31-34]. Even though a number of studies discuss the selection of reference genes [35-39] no study discussed circadian experiments and only one past study evaluated different mouse strains [40]. The majority of circadian studies still perform normalization using a single reference gene [41-44].

We evaluate ten candidate reference genes for their expression stability in a circadian experiment with mouse liver and adrenal glands in three mouse strains (C57BL/6JOlaHsd, 129Pas plus C57BL/6J and Crem $\mathrm{KO})$. Seven genes from our study have been evaluated previously under a variety of experimental conditions $[20,45,46]$. Three additional genes (Eif2a, Tbcc and Utp $6 c$ ) were selected by RefGene as the most stably expressed after the meta analysis of liver Affymetrix expression profile [25]. Publicly available microarray data have been successfully used before for reference gene selection $[47,48]$. We show that irrespective of the analysis applied (geNorm or NormFinder), different mouse strains show different rankings of reference genes. Utp $6 c$ was indicated as unstable reference gene in C57BL/6JOlaHsd livers by both programs (Figure 2A, Table 4A), whereas in the 129Pas plus C57BL/6J and Crem $\mathrm{KO}$ it ranked among the top three (Figure $2 \mathrm{C}$ and 2E; Table 4C, E).

When comparing gene expression between different mouse strains, three reference genes from the Affymetrix meta analyses ranked among the most stable, with Eif2a being first by both programs (Figure $2 \mathrm{G}$ and Table $4 G)$. This indicates that RefGenes tool is useful in narrowing down the number of candidate reference genes, especially when comparing mouse strains.

Differences in ranking of reference genes were observed not only between mouse strains, but also between tissues, as confirmed by both programs. Genes most stable in livers of 129Pas plus C57BL/6J and Crem $\mathrm{KO}$ strains ranked among the least stable in C57BL/ 6JOlaHsd mice (Figure 2 and Table 4). Adrenals show similar ranking between strains. Ppib is usually the most stable and Hprt1 the least stable gene in all strains by both programs. Our study shows that reference genes suitable for one mouse strain should not automatically be used for normalization in another strain. The same applies for different tissues.

The average expression stability value $M$ determined by geNorm shows little variability in gene expression between samples taking into account circadian effect both in liver and adrenals within each strain. This is in line with the pairwise variation value (V2/3), which is well below the 0.15 threshold value set by Vandensompele [20], indicating that normalization of target genes with a combination of the two best genes is sufficient. The $M$ value of reference genes was substantially higher when searching for stable genes between mouse stains. Here $A c t b$ was identified as the least stable in both liver and adrenals (Figure 2G,2H and Table 4G, H). Similar results were obtained when comparing human tissue samples [20].

To test whether selection of the least stable reference gene $(A c t b)$ affects normalization when compared to normalization with the two most stable genes selected by geNorm and NormFinder, a known and robust liver circadian gene $D b p$ has been normalized to three factors (Figure 4). Normalization to $A c t b$ leads to a large difference in the expression pattern between the C57BL/ 6JOlaHsd strain and the 129Pas plus C57BL/6J and Crem KO strains (Figure 4A). This can lead to a false impression that expression of $D b p$ is substantially higher in C57BL/6JOlaHsd. This difference, however, almost disappears when normalisation is performed on the two most stable genes Eif2a and Tbcc (geNorm) or Eif2a and Rn18 s (NormFinder) (Figure 4B and 4C). Even though the programs did not select identical reference genes, a similar $D b p$ expression profile was obtained in both cases. However, the ability of NormFinder to distinguish between different time points provides an advantage over geNorm when sufficient samples and genes are evaluated [22], as is the case in circadian experiments.

\section{Conclusions}

In this study we investigated the most reliable reference genes for normalization of circadian studies within or between mouse strains in livers and adrenal glands. The study is unique in its analysis of 3 mouse strains, 2 tissues and circadian sampling and in the magnitude of samples and genes tested (10 candidate reference genes in 228 samples by two programs). We show that differences in the reference genes exist between mouse strains as well as between tissues of the same strain. We also show that selection of a reference gene that appears stable in each mouse strain separately, can lead to interpretation errors, when used for normalization in different mouse stains. We identified altrernative reference genes that are stable in mouse strain comparisons. 


\section{Methods}

\section{Animals}

54 wild type (129Pas plus C57BL/6J) and $45 \mathrm{Crem}$ knock-out (Crem $\mathrm{KO})$ mice of the mixed strain and 35 mice of the inbred strain (C57BL/6JOlaHsd) were used. Animals had free access to food (Harland Tekland 2916) and water and were maintained under a 12:12 h light cycle (light on at 7:00 am, light off at 7:00 pm). The experiment was approved by the Veterinary Administration of the Republic of Slovenia (license number 34401$9 / 2008 / 4$ ) and was conducted in accordance with the European Convention for the protection of vertebrate animals used for experimental and other scientific purposes (ETS 123) as well as in accordance with National Institutes of Health guidelines for work with laboratory animals.

\section{Tissue samples}

Mice were sacrificed with cervical dislocation under dim red light every $4 \mathrm{~h}$ over a $24 \mathrm{~h}$ period starting on the second day after being transferred to dark: dark (DD) conditions. Immediately after they were sacrificed, liver and adrenal glands were excised, snap frozen in liquid $\mathrm{N}_{2}$ and stored at $-80^{\circ} \mathrm{C}$ for subsequent analysis. 96 liver and 58 adrenal glands from the mixed strain and 35 liver and 34 adrenal glands from the inbred strain were used for cDNA synthesis. The lower number of adrenal glands is due to insufficient amount of material or due to small RNA isolation yield. RNA was isolated from 30 $\mathrm{mg}$ of liver tissue and one adrenal gland per animal.

\section{RNA extraction and CDNA preparation}

Liver and adrenal gland samples were homogenized in $1000 \mu \mathrm{l}$ and $500 \mu \mathrm{l}$ of TRI reagent (Sigma) respectively and total RNA was isolated according to the manufactures instructions. RNA from liver samples of the inbred strain was isolated using QuickGene-810 (FujiFilm) according to manufacturer's instructions. RNA quantity and quality were assessed with NanoDrop and Agilent 2100 Bioanalyzer instruments. DNAse treatment was performed on all samples using DNAse I (Roche Applied Bioscience) according to the manufacturer's instructions. cDNA synthesis was carried out using SuperScript III reverse transcriptase (Invitrogen). $3 \mu \mathrm{g}$ of liver RNA was mixed together with $20 \mu \mathrm{l}$ of reverse transcriptase master mix which contained $8 \mu \mathrm{l}$ of $5 \times$ first strand buffer, $2 \mu \mathrm{l}$ of $100 \mathrm{mM}$ DTT, $2 \mu \mathrm{l}$ of $10 \mathrm{mM}$ $\mathrm{dNTP}$ mix, $1 \mu \mathrm{l}$ of random primers (Promega $500 \mathrm{ng} /$ $\mu \mathrm{l}), 0.75 \mu \mathrm{l}$ of SuperScript III $(200 \mathrm{U} / \mu \mathrm{l}), 0.75 \mu \mathrm{l}$ of RNAse OUT (Invitrogen) and $5.5 \mu \mathrm{l}$ of RNAse free water in a final volume of $40 \mu \mathrm{l} .1 \mu \mathrm{g}$ of adrenal gland RNA was mixed together with $10 \mu \mathrm{l}$ of reverse transcriptase master mix which contained $5 \mu$ l of $5 \mathrm{x}$ first strand buffer, $1.25 \mu \mathrm{l}$ of $100 \mathrm{mM}$ DTT, $1.25 \mu \mathrm{l}$ of $10 \mathrm{mM}$ dNTP mix, $0.65 \mu$ lof random primers (Promega $500 \mathrm{ng} / \mu \mathrm{l}), 0.5 \mu \mathrm{l}$ of SuperScript III $(200 \mathrm{U} / \mu \mathrm{l}), 0.5 \mu \mathrm{l}$ of RNAse OUT (Invitrogen) and $0.85 \mu \mathrm{l}$ of RNAse free water in a final volume of $25 \mu \mathrm{l}$. The reaction mixtures were incubated at $25^{\circ} \mathrm{C}$ for 5 minutes, $50^{\circ} \mathrm{C}$ for $60 \mathrm{~min}$ utes and $70^{\circ} \mathrm{C}$ for 10 minutes.

\section{Primer design}

Wherever possible, intron spanning primers for ten candidate reference genes were designed based on publicly available sequences (Table 2). Genes chosen belong to different functional classes, which reduces the chance of co-regulation. Primer specificity and amplification efficiency were also validated empirically with melting curve and standard curve analysis of a six fold dilution series.

\section{Quantitative qPCR}

Real time quantitative PCR was performed in a 384 well format on LightCycler 480 (Roche Applied Science) using LightCycler 480 SYBR Green I Master (Roche Applied Science). The PCR reaction consisted of $2.5 \mu \mathrm{l}$ of SYBR Green I Master, $1.15 \mu \mathrm{l}$ of RNAse free water, $0.6 \mu \mathrm{l}$ of $300 \mathrm{nM}$ primer mix and $0.75 \mu \mathrm{l}$ of cDNA in a total volume of $5 \mu \mathrm{l}$. Three technical replicates were performed for each sample. Cycling conditions were as follows: $10 \mathrm{~min}$ at $95^{\circ} \mathrm{C}$ followed by 40 rounds of $10 \mathrm{~s}$ at $95^{\circ} \mathrm{C}, 20 \mathrm{~s}$ at $60^{\circ} \mathrm{C}$ and $20 \mathrm{~s}$ at $72{ }^{\circ} \mathrm{C}$. Melting curve analysis for determining the dissociation of PCR products was performed from $65^{\circ} \mathrm{C}$ to $95^{\circ} \mathrm{C}$.

\section{Analysis of expression stability}

Expression stabilities of selected reference genes were evaluated by two publicly available programs, geNorm VBA and NormFinder applets for Microsoft Excel. geNorm calculates the stability of selected reference genes according to the similarity of their expression profile by pair-wise comparison and calculates $M$ value, where the gene with the highest value is the least stable one. All calculations were performed on quantities, which were transformed from Cq values based on gene specific efficiencies [20]. NormFinder also requires quantities input, but calculates a gene-stability value with a mathematical model based on separate analysis of the sample subgroups and estimation of both intra-and intergroup variation in expression levels [22]. Relative quantities (Q) needed for the input were calculated via the delta-Ct method with the formula $\mathrm{Q}=(\mathrm{E})^{\Delta \mathrm{Cq}} . \Delta \mathrm{Cq}$ equals $\mathrm{Cq}$ of the sample with the lowest $\mathrm{Cq}$ value (highest abundance) minus Cq of a sample. Efficiency (E) corrected relative amount were calculated. Normalization factors were calculated based on geometric averaging of the 
most stable reference genes. Normalization was carried out by dividing relative quantities with the normalization factor [20].

\section{Acknowledgements}

The work was supported by the Slovenian Research Agency Grants J1-9438 and P1-0527. Rok Kosir was supported by the fellowship from the Slovenian Research Agency. We thank Jernej Ule for helpful discussions and careful reading of the manuscript.

\section{Author details}

${ }^{1}$ Center for Functional Genomics and Bio-Chips, Institute of Biochemistry, Faculty of Medicine, University of Ljubljana, Zaloska 4, SI-1000 Ljubljana, Slovenia. ${ }^{2}$ Institute of Biochemistry, Faculty of Medicine, University of Ljubljana, Vrazov trg 2, SI-1000 Ljubljana, Slovenia. ${ }^{3}$ Medical Experimental Centre, Institute of Pathology, Faculty of Medicine, University of Ljubljana, Zaloska 4, SI-1000 Ljubljana, Slovenia. ${ }^{4}$ Center for Animal Genomics, Veterinary Faculty; University of Ljubljana, Gerbiceva 60, SI-1000 Ljubljana, Slovenia. ${ }^{5}$ University Medical Center Ljubljana, Department of Haematology, Zaloska cesta 7, SI-1000 Ljubljana, Slovenia.

\section{Authors' contributions}

RK carried out qPCR and data analysis and wrote the manuscript. JA and MG participated in the planning, sampling and data analysis. MP and GM are responsible for animal experiments and participated in sampling, design of the study and provided useful discussions. MF participated in study design, data analysis and provided useful discussion.DR supervised the study, participated in study design and coordination. All authors read and approved the final manuscript.

Received: 13 April 2010 Accepted: 16 August 2010

Published: 16 August 2010

\section{References}

1. Ko CH, Takahashi JS: Molecular components of the mammalian circadian clock. Hum Mol Genet 2006, 15(Spec No 2):R271-277.

2. Lowrey PL, Takahashi JS: Mammalian circadian biology: elucidating genome-wide levels of temporal organization. Annu Rev Genomics Hum Genet 2004, 5:407-441.

3. Reppert SM, Weaver DR: Coordination of circadian timing in mammals. Nature 2002, 418(6901):935-941.

4. Ishida N: Circadian clock, cancer and lipid metabolism. Neurosci Res 2007, 57(4):483-490.

5. Sahar S, Sassone-Corsi P: Metabolism and cancer: the circadian clock connection. Nat Rev Cancer 2009, 9(12):886-896.

6. Li S, Lin JD: Molecular control of circadian metabolic rhythms. J App/ Physiol 2009, 107(6):1959-1964.

7. Turek FW, Joshu C, Kohsaka A, Lin E, Ivanova G, McDearmon E, Laposky A Losee-Olson S, Easton A, Jensen DR, Eckel RH, Takahashi JS, Bass J: Obesity and metabolic syndrome in circadian Clock mutant mice. Science 2005, 308(5724):1043-1045.

8. Antoch MP, Gorbacheva WY, Vykhovanets O, Toshkov IA, Kondratov RV, Kondratova AA, Lee C, Nikitin AY: Disruption of the circadian clock due to the Clock mutation has discrete effects on aging and carcinogenesis. Cell Cycle 2008, 7(9):1197-1204.

9. Rudic RD, McNamara P, Curtis AM, Boston RC, Panda S, Hogenesch JB, Fitzgerald GA: BMAL1 and CLOCK, two essential components of the circadian clock, are involved in glucose homeostasis. PLOS Biol 2004, 2(11):e377.

10. Vitaterna MH, King DP, Chang AM, Kornhauser JM, Lowrey PL, McDonald JD, Dove WF, Pinto LH, Turek FW, Takahashi JS: Mutagenesis and mapping of a mouse gene, Clock, essential for circadian behavior. Science 1994, 264(5159):719-725.

11. Oishi K, Miyazaki K, Ishida N: Functional CLOCK is not involved in the entrainment of peripheral clocks to the restricted feeding: entrainable expression of mPer2 and BMAL1 mRNAs in the heart of Clock mutant mice on Jcl:ICR background. Biochem Biophys Res Commun 2002, 298(2):198-202.
12. Yoshiki A, Moriwaki K: Mouse phenome research: implications of genetic background. ILAR J 2006, 47(2):94-102.

13. Fleming RE, Holden CC, Tomatsu S, Waheed A, Brunt EM, Britton RS, Bacon BR, Roopenian DC, Sly WS: Mouse strain differences determine severity of iron accumulation in Hfe knockout model of hereditary hemochromatosis. Proc Natl Acad Sci USA 2001, 98(5):2707-2711.

14. Montagutelli $X$ : Effect of the genetic background on the phenotype of mouse mutations. J Am Soc Nephrol 2000, 11(Suppl 16):S101-105.

15. Wahlsten D, Bachmanov A, Finn DA, Crabbe JC: Stability of inbred mouse strain differences in behavior and brain size between laboratories and across decades. Proc Natl Acad Sci USA 2006, 103(44):16364-16369.

16. Bustin SA, Nolan T: Pitfalls of quantitative real-time reverse-transcription polymerase chain reaction. J Biomol Tech 2004, 15(3):155-166.

17. Huggett J, Dheda K, Bustin S, Zumla A: Real-time RT-PCR normalisation; strategies and considerations. Genes Immun 2005, 6(4):279-284.

18. Bustin S: Quantification of mRNA using real-time reverse transcription PCR (RT-PCR): trends and problems. J Mol Endocrinol 2002, 29(1):23-39.

19. Bustin SA, Benes $V$, Nolan T, Pfaffl MW: Quantitative real-time RT-PCR-a perspective. J Mol Endocrinol 2005, 34(3):597-601.

20. Vandesompele J, De Preter K, Pattyn F, Poppe B, Van Roy N, De Paepe A, Speleman F: Accurate normalization of real-time quantitative RT-PCR data by geometric averaging of multiple internal control genes. Genome Biol 2002, 3(7):RESEARCH0034.

21. Pfaffl MW, Tichopad A, Prgomet C, Neuvians TP: Determination of stable housekeeping genes, differentially regulated target genes and sample integrity: BestKeeper-Excel-based tool using pair-wise correlations. Biotechnol Lett 2004, 26(6):509-515.

22. Andersen $C L$, Jensen $J L$, Orntoft TF: Normalization of real-time quantitative reverse transcription-PCR data: a model-based variance estimation approach to identify genes suited for normalization, applied to bladder and colon cancer data sets. Cancer Res 2004, 64(15):5245-5250.

23. Radonic A, Thulke S, Mackay IM, Landt O, Siegert W, Nitsche A: Guideline to reference gene selection for quantitative real-time PCR. Biochem Biophys Res Commun 2004, 313(4):856-862

24. Dheda K, Huggett JF, Bustin SA, Johnson MA, Rook G, Zumla A: Validation of housekeeping genes for normalizing RNA expression in real-time PCR. Biotechniques 2004, 37(1):112-114, 116, 118-119.

25. Hruz T, Laule O, Szabo G, Wessendorp F, Bleuler S, Oertle L, Widmayer P, Gruissem W, Zimmermann P: Genevestigator V3: a reference expression database for the meta-analysis of transcriptomes. Advances in Bioinformatics 2008, 420747:5.

26. Wuarin J, Schibler U: Expression of the liver-enriched transcriptional activator protein DBP follows a stringent circadian rhythm. Cell 1990, 63(6):1257-1266

27. Lopez-Molina L, Conquet F, Dubois-Dauphin M, Schibler U: The DBP gene is expressed according to a circadian rhythm in the suprachiasmatic nucleus and influences circadian behavior. EMBO J 1997, 16(22):6762-6771.

28. VanGuilder HD, Vrana KE, Freeman WM: Twenty-five years of quantitative PCR for gene expression analysis. Biotechniques 2008, 44(5):619-626.

29. Bustin SA: Real-time, fluorescence-based quantitative PCR: a snapshot of current procedures and preferences. Expert Rev Mol Diagn 2005, 5(4):493-498.

30. Dheda K, Huggett JF, Chang JS, Kim LU, Bustin SA, Johnson MA, Rook GA, Zumla $A$ : The implications of using an inappropriate reference gene for real-time reverse transcription PCR data normalization. Anal Biochem 2005, 344(1):141-143

31. Takahashi JS, Hong HK, Ko CH, McDearmon EL: The genetics of mammalian circadian order and disorder: implications for physiology and disease. Nat Rev Genet 2008, 9(10):764-775.

32. Green CB, Takahashi JS, Bass J: The meter of metabolism. Cell 2008 134(5):728-742.

33. Levi F, Okyar A, Dulong S, Innominato PF, Clairambault J: Circadian timing in cancer treatments. Annu Rev Pharmacol Toxicol 50:377-421.

34. Levi F, Schibler U: Circadian rhythms: mechanisms and therapeutic implications. Annu Rev Pharmacol Toxicol 2007, 47:593-628.

35. Cook NL, Kleinig TJ, van den Heuvel C, Vink R: Reference genes for normalising gene expression data in collagenase-induced rat intracerebral haemorrhage. BMC Mol Biol 11:7. 
36. Maroufi A, Van Bockstaele E, De Loose M: Validation of reference genes for gene expression analysis in chicory (Cichorium intybus) using quantitative real-time PCR. BMC Mol Biol 11:15.

37. Langnaese K, John R, Schweizer H, Ebmeyer U, Keilhoff G: Selection of reference genes for quantitative real-time $P C R$ in a rat asphyxial cardiac arrest model. BMC Mol Biol 2008, 9:53.

38. Kessler $Y$, Helfer-Hungerbuehler AK, Cattori V, Meli ML, Zellweger B, Ossent $P$, Riond B, Reusch CE, Lutz H, Hofmann-Lehmann R: Quantitative TaqMan real-time PCR assays for gene expression normalisation in feline tissues. BMC Mol Biol 2009, 10:106.

39. Saviozzi S, Cordero F, Lo lacono M, Novello S, Scagliotti GV, Calogero RA: Selection of suitable reference genes for accurate normalization of gene expression profile studies in non-small cell lung cancer. BMC Cancer 2006, 6:200.

40. Frericks $M$, Esser C: A toolbox of novel murine house-keeping genes identified by meta-analysis of large scale gene expression profiles. Biochim Biophys Acta 2008, 1779(12):830-837.

41. Asher G, Gatfield D, Stratmann M, Reinke H, Dibner C, Kreppel F, Mostoslavsky R, Alt FW, Schibler U: SIRT1 regulates circadian clock gene expression through PER2 deacetylation. Cell 2008, 134(2):317-328.

42. Canaple L, Rambaud J, Dkhissi-Benyahya O, Rayet B, Tan NS, Michalik L, Delaunay F, Wahli W, Laudet V: Reciprocal regulation of brain and muscle Arnt-like protein 1 and peroxisome proliferator-activated receptor alpha defines a novel positive feedback loop in the rodent liver circadian clock. Mol Endocrinol 2006, 20(8):1715-1727.

43. Liu AC, Tran HG, Zhang EE, Priest AA, Welsh DK, Kay SA: Redundant function of REV-ERBalpha and beta and non-essential role for Bmal1 cycling in transcriptional regulation of intracellular circadian rhythms. PLoS Genet 2008, 4(2):e1000023.

44. Ptitsyn AA, Zvonic S, Conrad SA, Scott LK, Mynatt RL, Gimble JM: Circadian clocks are resounding in peripheral tissues. PLoS Comput Biol 2006, 2(3): e16.

45. Shi G, Zhang Z, Feng D, Xu Y, Lu Y, Wang J, Jiang J, Li X, Ning G: Selection of reference genes for quantitative real-time reverse transcriptionpolymerase chain reaction in concanavalin A-induced hepatitis model. Anal Biochem

46. Tatsumi K, Ohashi K, Taminishi S, Okano T, Yoshioka A, Shima M: Reference gene selection for real-time RT-PCR in regenerating mouse livers. Biochem Biophys Res Commun 2008, 374(1):106-110.

47. Popovici V, Goldstein DR, Antonov J, Jaggi R, Delorenzi M, Wirapati P. Selecting control genes for RT-QPCR using public microarray data. BMC Bioinformatics 2009, 10:42.

48. Shulzhenko N, Yambartsev A, Goncalves-Primo A, Gerbase-DeLima M, Morgun A: Selection of control genes for quantitative RT-PCR based on microarray data. Biochem Biophys Res Commun 2005, 337(1):306-312.

doi:10.1186/1471-2199-11-60

Cite this article as: Kosir et al:: Determination of reference genes for circadian studies in different tissues and mouse strains. BMC Molecular Biology 2010 11:60

\section{Submit your next manuscript to BioMed Central and take full advantage of:}

- Convenient online submission

- Thorough peer review

- No space constraints or color figure charges

- Immediate publication on acceptance

- Inclusion in PubMed, CAS, Scopus and Google Scholar

- Research which is freely available for redistribution

Submit your manuscript at www.biomedcentral com/submit
C Biomed Central 